\title{
Jóvenes romaníes en asentamientos chabolistas: movilidad y contextos de exclusión en España y Francia
}

\section{Young romans in shanty town settlements: mobility and contexts of exclusion in Spain and France}

\author{
José David Gutiérrez Sánchez \\ Universidad Pablo de Olavide (España) \\ jdgutsan@upo.es \\ Chabier Gimeno Monterde \\ Universidad de Zaragoza (España) \\ chabierg@unizar.es
}

Fecha de recepción: 04/12/2018

Fecha de aceptación: 13/09/2019

\section{Resumen}

Este artículo de corte cualitativo tiene como objetivo realizar una investigación evaluativa y analítica de la intervención social con adolescentes de procedencia rumana y etnia gitana en los asentamientos de El Gallinero (Madrid) y Thiersbenauge (Burdeos), con el fin de establecer estrategias para proyectos de Trabajo Social con dichos colectivos. Los hallazgos han posibilitado por un lado, el conocimiento de una población estigmatizada y que vive en condiciones de vulnerabilidad social en la periferia de grandes ciudades, y por otro, el papel trascendental que alberga la movilidad laboral para dicha población. Promover un enfoque de intervención integral donde se creen redes colaborativas entre las administraciones públicas, las entidades sociales y la propia población, sería un modo de avanzar en la igualdad de oportunidades sobre todo en etapas como la adolescencia.

Palabras clave: Romaníes; Exclusión social; Adolescencia; Movilidad; Francia; España.

\footnotetext{
Abstract

This qualitative article aims to conduct an evaluative and analytical investigation of the social intervention with Romanian and gypsy teenagers in the settlements of El Gallinero (Madrid) and Thiers-Benauge (Bordeaux), in order to establish strategies for Social Work projects with these groups. The findings have made possible, on the one hand, the knowledge of a stigmatized population living in conditions of social vulnerability in the periphery of large cities, and on the other, the transcendental role that houses labor mobility for said population. Promoting
} 
Jóvenes romaníes en asentamientos chabolistas... - J.D. Gutiérrez y G. Gimeno

a comprehensive intervention approach where collaborative networks are created between public administrations, social entities and the population itself, would be a way to advance equality of opportunities especially in stages such as adolescence.

Keywords: Roma; Social exclusion; Adolescence; Mobility; France; Spain

\section{INTRODUCGIÓN}

Este trabajo tiene por objetivo realizar una investigación evaluativa y analítica de la intervención social con adolescentes de procedencia rumana y etnia gitana con el fin de establecer estrategias para proyectos de Trabajo Social con dichos colectivos. Los procesos de exclusión social y pobreza que vive la población romaní en Europa se convierten en un hándicap importante que afecta a la inclusión social, acceso al mercado de trabajo, hábitat y al contexto social y de relaciones con la sociedad autóctona del lugar donde se asienta dicha población provocando, en la mayoría de los casos, una movilidad constante entre países como España y Francia.

Con el objeto de fundamentar esta investigación sobre la intervención social de los jóvenes romaníes se han empleado técnicas de observación participante y entrevista directamente en el contexto social y comunitario donde se asentaba la población objeto de estudio. La investigación se ha llevado a cabo en dos periodos de tiempo. El primero de ellos entre 2010-2014 cuándo se llevó a cabo el trabajo de campo en dos asentamientos, por un lado en el denominado asentamiento chabolista de El Gallinero (Madrid) durante un año, y por otro el asentamiento de Thiersbenauge (Burdeos) a lo largo de cuatro meses. El segundo periodo de investigación se desarrolló entre 2015-2017. En este periodo de tiempo se analizaron los datos extraídos y se obtuvieron, partiendo de la experiencia empírica de la etapa anterior, propuestas de intervención social y además, se analizaron las causas de la movilidad y los medios se subsistencia empleados por los romaníes durante la estancia en contextos de gran vulnerabilidad social como son los asentamientos chabolistas en las periferias de grandes ciudades. Estos resultados nos conducen a tener en cuenta las causas originarias de la movilidad y los procesos de adaptación tanto en España como en Francia.

\subsection{Inicio de la movilidad en España y Francia}

Al observar la movilidad de diferentes colectivos dentro de la Unión Europea en tiempos contemporáneos, bajo un emplazamiento dónde lo líquido (Bauman, 2006) se torna incuestionable y dónde las respuestas tomadas por parte de los Estados adquieren diferentes tintes (Ferrera, 2018), es oportuno observar cómo un amplio sector de la población comunitaria se traslada de forma constante en busca de mejores respuestas a las necesidades y demandas que la pobreza expresa (Krmpotic, 1999), 


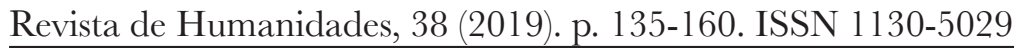

y en concreto cuando de juventud se habla. La Europa del Siglo XXI manifiesta una nueva constelación de conflictos, que pasan desde las tensiones internas del Estado de Bienestar de cada país a incluso la propia integración europea de los mismos, donde se pone en valor el libre movimiento, la ley de competencia, la creación del mercado único y la unión monetaria (Ferrera, 2005 y 2018).

En los últimos 10 años se ha incrementado el interés por observar la situación que el colectivo rumano y de etnia gitana vive en el contexto español y francés. En este sentido, es posible diferenciar procesos distintos en ambos países (desde la caída del régimen de Nicolae Ceaucescu en 1999) ya que Rumanía avanzó a una transición política y económica, produciendo cambios profundos en la sociedad y dando lugar a uno de los casos de mayor empobrecimiento de la Europa del Este (Pajares, 2007).

Los primeros grupos numerosos de gitanos rumanos que llegaron a España lo hicieron a finales de los años noventa a la ciudad de Madrid (Gutiérrez, 2017; Las Heras, 1999). En el contexto francés y según Reyners (1995) en Francia hubo dos momentos destacados de llegadas de romaníes, por un lado entre 1980 y 1981 y por otro entre 1987 y 1988 (Pajares, 2007) fruto de continuas regulaciones internas en relación al mercado laboral europeo. A modo de síntesis distinguimos tres periodos; por un lado los años 80 destacado sobre todo por la reactivación de la economía en la Europa Occidental (Pajares, 2007) que marca la demanda de mano de obra motivando por tanto los flujos migratorios; posteriormente le precede la década de los 90 señalada por la caída del muro de Berlín y la apertura de Rumanía, donde distinguimos movimientos con el componente de reagrupación familiar (donde se observa bastante población que llega a Alemania y Polonia) pero además, el carácter intermitente de dichos flujos y la aparición de otros destinos como Italia, Portugal y España; por último la década de los 2000 marcada sobre todo por la ampliación de la Unión Europea en 2004 y la entrada de Rumanía en 2007. Éste periodo de tiempo hasta nuestros días ha cambiado la interpretación de las migraciones, convirtiéndose los desplazamientos en movilidad por ser ya ciudadanos europeos (Marcu, 2013).

En la actualidad, se muestra relevante analizar los procesos de exclusión que vienen desarrollándose en torno al colectivo romaní (Gutiérrez y Ortega, 2019). Según Castel (1995), ni la integración ni la exclusión son estados inamovibles, sino la expresión extrema de un proceso. Por lo tanto, es pertinente indagar en los procesos de exclusión del colectivo en cuestión, ya que de esta forma se podrán obtener respuestas que ayuden o incidan en la inclusión social, sobre todo para la juventud e infancia.

En definitiva, todo el proceso de ampliación europea y el desarrollo de tratados, han impactado con una crisis económica sin precedentes, lo cual sobre todo, ha causado en la juventud y en sus expectativas de encontrar un empleo (autóctona pero sobre todo del Este), una situación compleja (Marcu, 2018) que ha evolucionado en determinados casos en más exclusión y segregación social. 


\section{MARCO TEÓRICO}

2.1 Pobreza infantil y movilidad de los Jóvenes gitanos.

Múltiples han sido los autores que han abordado la cuestión Romaní o Rrom (como se conoce en Europa a los gitanos procedentes del Este) desde diversas perspectivas; en cuanto a la exclusión social (Di Noia, 2016), persecución del Pueblo Gitano (Brearley, 2001), identidad (Bontempelli, 2015; Csepeli y Simon, 2004), por identificación como minorías (Vassilev, 2004), razones ecológicas y económicas (Griffin, 2008; Pajares, 2007; López-Riopedre, 2017; Viruela, 2006), y vivir en comunidades segregadas (Clave-Mercier y Olivera, 2018; Gutiérrez, 2015; Harper, Steger y Filcak, 2009).

En la actualidad existe un interés destacado en profundizar éste colectivo pero apoyados en la noción de movilidad (Cresswell, 2006; Marcu, 2013; Sheller y Urry, 2006) y además desde la perspectiva de la juventud (Briggs, 2016; Gutiérrez, 2017; Marcu, 2018; Humphris y Sigona, 2016).

En relación a la juventud, Vigh (2006) explora la conexión entre juventud y movimiento incorporando la terminología navegación social. Vigh desarrolló la idea de navegación social partiendo de la teoría práctica y la noción de oportunidad de vida que tiene como impulsor a Dahrendorf (1979). La navegación social como concepto "es una forma de la agencia que supone la habilidad de actuar en relación a las restricciones y posibilidades, asi como la habilidad de trazar y actualizar uno de los movimientos presentes hacia un futuro imaginado" (Marcu, 2018, p. 33).

Frecuentemente, el acto de moverse entre dos espacios dentro de la movilidad en etapas como infancia y adolescencia, ha sido un recurso que se ha distinguido por representar cierto estatus y poder, sobre todo en grupos sociales privilegiados (Marcu, 2018; Sheller y Urry, 2006). Sin embargo, la apertura del espacio europeo con su ampliación de la Unión Europea hacia el Este ha proporcionado un cambio en la tendencia y es observable cómo un gran número de niños y niñas, independientemente de su posición social, transitan por el espacio europeo acompañados de sus familias, en la mayoría de los casos (Grill, 2012). El origen de la movilidad de jóvenes gitanos guarda, sobre todo, un componente económico con el desempleo (en origen) como denominador común (Diminescu, 2004). En el caso de chicos y chicas que se movilizan con sus familias, el factor económico depende de la capacidad económica del propio grupo familiar, aunque en aquellos casos en que los jóvenes practican la movilidad, éstos suelen buscar alternativas a la pobreza en otros países asumiendo el papel responsable de buscar recursos para posteriormente ser enviados a origen (Gimeno, 2013).

La población gitana constituye una galaxia de minorías que según Dell'Agnese y Vitale (2007) y Baldoni (2012) no son para nada homogéneas desde la perspectiva histórica, cultural y religiosa. Hablamos de segmentos étnicos que individualmente 


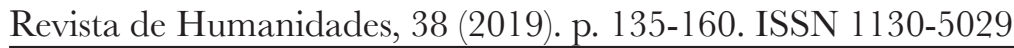

presentan una visión de la movilidad propia. Lo cual hace que determinados comportamientos en casos concretos sean más complejos de observar. A pesar de la complejidad, se establecen unos mínimos que acompañan con carácter común y general a aquellas personas que emprenden el movimiento. Según Kholi, "el movimiento a lo largo del tiempo y a través de los territorios guarda relación con la madurez de la persona" (2014, p. 33). Es decir, la vivencia que manifiesta la persona interiormente (o el grupo) en su proceso móvil en relación al aprendizaje vivido (siendo positivo o negativo) hace que articule un bagaje que innegablemente perturba al comportamiento (tanto presente como futuro) y que posteriormente exterioriza y se hace palpable en la sociedad, induciendo estados o momentos que pueden ser aceptados o no socialmente (Gutiérrez, 2017).

Por otro lado, la movilidad de jóvenes también puede estar relacionada (aunque no necesariamente) con el riesgo. Entendemos el riesgo como concepto multifacético y dinámico (Stevenson, 1999; Ryan, 1996; Warner, 1992). El riesgo incorpora cierta probabilidad de que se desarrollen determinadas situaciones que pueden desarrollar el mismo. Para llegar a interpretar el riesgo debe tenerse en cuenta que la realidad y los fenómenos que interaccionan no son independientes (Luhmann, 1991), es decir, que existe una relación entre ellos. Por lo tanto se muestra relevante conocer el contexto y aquellos aspectos y fenómenos que catalizan o mantienen el riesgo dentro de las sociedades.

La sociedad del riesgo es un concepto abordado ampliamente por Ulrich Beck (1992) el cual se basa, como sostiene Climent (2006), en la constatación de que en las sociedades presentes la producción social de riqueza va acompañada metódicamente por una progresiva producción social del riesgo.

\subsection{Comunidad e intervención}

En los últimos años se han ido desarrollando numerosos asentamientos chabolistas de población rumana y etnia gitana en las periferias de las grandes ciudades europeas. En España podemos encontrar casos en la ciudad de Madrid como el poblado chabolista de El Gallinero (Gutiérrez, 2015), y en el área Metropolitana de Barcelona (López, 2014). En el contexto francés es destacado también la región de Aquitania donde en la ciudad de Burdeos podemos localizar el asentamiento de Thiers-benauge (Gutiérrez, 2017a) entre otros.

Estos asentamientos han soportado las intervenciones sociales que se han ido desarrollando por medio de la administración pública y entidades privadas con el interés de mejorar la situación de inestabilidad, en cuanto insalubridad, habitalidad y movilidad de personas. La continua persistencia en la implantación de intervenciones sociales (a veces favorables para la inclusión social y otras desfavorables por mantenimiento en el tiempo de la exclusión) han desencadenado un escenario de 
imposición de patrones vitales por parte de los grupos dominantes o sociedades autóctonas y la aceptación de su situación, de parte de los excluidos (Dovigo, 2014).

En este sentido el Trabajo Social Comunitario ha tenido un papel trascendental a la hora de abordar situaciones complejas tanto por el contexto como por las propias personas (beneficiarias como profesionales de la intervención). A lo largo del Siglo XX, numerosos autores han intentado plasmar una definición de Trabajo Social Comunitario (Friedlander, 1977; Marchioni, 1999; McNeil, 1954; Moix, 1991; Twelvetrees, 1985). El Trabajo Social Comunitario es una unidad de intervención que tiene como objetivo mejorar las comunidades por medio de la acción colectiva (Twelvetrees, 1985), donde destaca la participación de todas sus partes estableciendo un objetivo u objetivos comunes.

El trabajo con la comunidad y en concreto en contextos de riesgo, nos permite determinar las necesidades de una población en concreto, lo cual permite incidir positivamente sobre dichas necesidades y carencias detectadas. Teniendo en cuenta los estudios de Pastor (2015) y Picornell (2015), hablamos de un trabajo social con comunidades centrado en el modelo de desarrollo local estratégico, integral y orientado hacia el empowerment comunitario (Gaitán, 2003; Gutiérrez y Diz, 2017; Pastor, 2004). El Trabajo Social Comunitario además nos permite observar otros elementos estructurales y propios de la comunidad como son el territorio, población, demanda y recursos existentes (Marchioni, 1989).

\subsection{Pobreza infantil en España y Europa}

En 2017, La Red Europea de Lucha contra la Pobreza y la Exclusión Social presentaba su séptimo informe anual de seguimiento del indicador AROPE (At Risk Of Poverty and/or Exclusion) en España y Europa, donde se ofrece una evaluación del grado de cumplimiento del objetivo social especificado en la Estrategia 2020 para el conjunto del Estado español, así como las consecuencias que la crisis económica y de las políticas sociales implementadas han tenido para la sociedad en el ámbito de la pobreza y/o exclusión social (EAPN, 2017).

Partiendo de la Estrategia 2020 y en el contexto español, el objetivo de reducción de personas en riesgo de pobreza está detallado en el Programa Nacional de Reformas (2011), el cual señala cómo España se marca el objetivo de reducir entre 1.400 .000 y 1.500 .000 (durante el periodo de 2009-2019) el número de personas en riesgo de pobreza y exclusión social de acuerdo con un indicador agregado que incluye a personas que viven por debajo del umbral de la pobreza (pobreza relativa); personas que sufren privación material severa y personas que viven en hogares con una intensidad de empleo baja o nula.

En 2016 España alcanzaba un 32.9\% de población infantil en riesgo de pobreza y exclusión social, situándose Francia en el 22.6\%. La media de la Unión Europea 


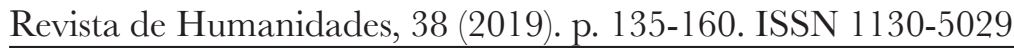

(UE28) alcanzaba un $26.4 \%$. Es decir, de un lado España se situaba como el quinto país de la Unión Europea con más pobreza infantil, siendo Francia dieciseisavos de la lista (ostentando Rumanía la cabeza de lista y Dinamarca la parte baja de la tabla). La "recuperación económica" en la Unión Europea no acompaña los indicadores de pobreza y exclusión social. Además en el periodo entre 2008-2013 los datos macroeconómicos señalaban como el gasto social en infancia en relación al PIB para España y Francia es 1.3 y 2.5\% respectivamente (Eurostat, 2014).

Estos datos referidos sobre todo a pobreza infantil, guardan directamente relación con la adolescencia. La OCDE (2010) señalaba como los jóvenes a pesar de poder tener calificaciones altas, están atrapados en una serie de puestos de trabajo precarios que son interrumpidos por períodos de desempleo o inactividad económica. Este patrón es aún más señalado en los países del Sur de Europa donde además podemos observar un alto grado de graduados universitarios (Marcu, 2018: Purcell, 2010). Estos datos son aún mayores en contextos de riesgo con pocos ingresos e inmersos en procesos de exclusión social (Gutiérrez, 2017a). Por último y teniendo en cuenta la tasa de desempleo, ésta incide directamente en la posibilidad de encontrar un empleo, ayudar a la familia, e incluso emprender una empresa o pagar unos estudios. Eurostat (2018) señala la media de desempleo en la Unión (UE28) en un 7\%, estando España en el $15.8 \%$ y Francia en el 9.2\%. En desempleo juvenil, España se sitúa en el $36,3 \%$ frente a un $16,8 \%$ en la UE.

\section{METODOLOGÍA}

La investigación, concebida como multisituada (Empez, 2009; Gimeno y Gutiérrez, 2019), fue llevada a cabo entre dos países, España y Francia. El objetivo principal consistió en realizar una investigación evaluativa y analítica de la intervención social con adolescentes de procedencia rumana y etnia gitana con el fin de establecer estrategias para proyectos de Trabajo Social con dichos colectivos. La investigación contó con dos periodos de tiempo concretos, 2010-2014 y 20152017. El primero de ellos entre 2010-2014 se llevó a cabo el trabajo de campo en dos asentamientos: por un lado en el asentamiento chabolista de El Gallinero (Madrid) durante un año de duración; y por otro el asentamiento de Thiers-benauge (Burdeos) a lo largo de cuatro meses. Para poder obtener una información primaria fiable y directa por parte de los sujetos del estudio, se accedió a ambos asentamientos por medio de la incorporación a las actividades de entidades sociales no lucrativas, siendo en Madrid la Fundación Imaginario Social y en Burdeos L'Assotiation Départamentale les Amis de Voyageurs de la Gironde (ADAV33), desde las cuales se obtuvo contacto con Servicios Sociales Comunitarios, Grupos locales y regionales orientados a la atención de población excluida, acceso a datos de regionales, contacto con diversos profesionales (Trabajadores Sociales, Educadores Sociales, Psicólogos, Maestros, Médicos, Enfermeros) y contacto con otras entidades sociales que 
operaban en ambos lugares. El motivo de analizar dos asentamientos en dos países distintos básicamente radica en la comprobación, en primer lugar, del desarrollo de mismas conductas y actividades por parte de la población ante casos de marginación y exclusión social, y en segundo lugar para observar cómo problemas con orígenes parecidos se intervienen de formas diversas.

En esta primera parte de la investigación se llevaron a cabo las técnicas de observación participante y entrevista. El análisis realizado en este trabajo ha estado vinculado al estudio del contexto social del colectivo Rrom en cuanto la gestión y acción publico-privada. En total se realizaron 86 entrevistas en profundidad, cubriendo por saturación de información, todos los informantes establecidos previamente como claves en el estudio preliminar. Las entrevistas se llevaron a cabo tanto en los asentamientos como fuera de ellos. La ubicación la marcaba la posibilidad de estar influenciados por el contexto social. En algunos casos se llevaron a cabo las entrevistas en parques y centros comerciales. Las entrevistas las clasificamos del siguiente modo: 9 entrevistas realizadas a miembros de entidades sociales (miembros de la Fundación Imaginario Social y ADAV33), 11 realizadas por medio del Proyecto PUCAFREU (Promoting Unprotected Unaccompained Children's Access to Fundamental Rights in the European Union) a menores entre 11 y 18 años en El Gallinero, 8 a informantes clave o líderes (investigadores y personas de otros países como Francia y Rumanía que destacaban por diversas iniciativas), 22 a familiares de los sujetos de la intervención (16 familiares en Madrid y 6 familiares en Burdeos), 31 a menores en colaboración con la Fundación Imaginario Social (entre 10 y 18 años, 23 en El Gallinero y 8 en Thiers-Benauge) y 5 entrevistas realizadas grupalmente por medio de las actividades en la Fundación Imaginario Social durante 2010-2012.

Acceder a menores de edad en posiciones vulnerables puede llegar a ser dificultoso (Kohli, 2006; Pastoor, 2015). Hay que especificar que las entrevistas realizadas a dichos menores se ubicaban dentro de las actividades de distensión y reuniones grupales que se llevaban a cabo desde las entidades sociales, es decir, el acceso a la información tenía un carácter ameno y no directo como se hizo con adultos. Por otro lado, es preciso entablar una relación de confianza con el colectivo gitano rumano previamente a la aplicación de las técnicas de investigación. Esto viene dado por la relación de desconfianza por parte de la población ante personas que puedan comprometer o alterar su día a día en los asentamientos, es decir, fue preciso en primer lugar hacer visitas, conocer poco a poco a la población generando confianza, conocer a las entidades sociales y sus profesionales (Parroquias, voluntarios, administración local y regional). Este trabajo previo de acercamiento ayudó posteriormente al desarrollo de la investigación.

El segundo periodo de investigación se desarrolló entre 2015-2017. En este periodo de tiempo se analizaron los datos extraídos. Estos resultados nos conducen a tener en cuenta las causas originarias de la movilidad y los procesos de adaptación 


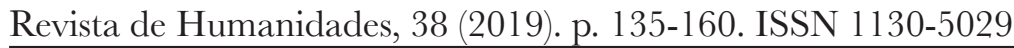

tanto en España como en Francia. El diagnóstico de la situación se abordó mediante análisis documental e información primaria. El análisis documental consistió en la revisión de la normativa vigente relativa a la gestión de este tipo de asentamientos, denuncias de entidades sociales y documentos elaborados por expertos.

El análisis de las entrevistas consistió en primer lugar en la trascripción de las mismas para posteriormente ordenar la información por medio de categorías de análisis donde destacamos (movilidad, exclusión, familia, comunidad, características sociodemográficas, proyectos). Posteriormente se codificó la información asignando unidades de información descriptivos. Por último se integró la información, relacionando las categorías entre sí con los fundamentos teóricos de la investigación.

\section{RESULTADOS Y ANÁLISIS}

4.1 Características sociodemográficas de menores y familias en los asentamientos

Los asentamientos de El Gallinero y Thiers-benauge de forma similar, forman parte de un proceso de movilidad transnacional marcada sobre todo por motivos de carácter económico dentro de la Unión Europea. Partiendo la experiencia en terreno durante 2010-2017, se constata como asentamientos ubicados distantes uno del otro, adquieren de forma parecida la misma estructura (arquitectónica) y de relaciones sociales entre sus habitantes. Por un lado, ambos lugares presentan una composición donde se ubican chabolas de aproximadamente 35 metros cuadrados sin acceso estable a electricidad, agua y saneamiento. En el caso de El Gallinero y teniendo en cuenta el último censo elaborado por la Agencia de Vivienda Social de la Comunidad de Madrid, la población residente es de 184 personas (integradas en 44 unidades familiares) de las cuales 103 son menores de edad. En Thiers-benauge hablamos de 220 personas donde aproximadamente el 50\% son niños y niñas (Gutiérrez, 2017a). Aunque el número de personas puede variar según el periodo de tiempo concreto que se observe, se verifica como dentro de las comunidades no existe una masculinización de la movilidad, es decir, el número de hombres y mujeres es parecido en ambos lugares representando aproximadamente un $56 \%$ los hombres y un $44 \%$ las mujeres aproximadamente.

"No quiero vivir aqui, las ratas son muy peligrosas, sobre todo por la noche. No podemos pagar el alquiler de una casa, es muy caro y somos muchos". Chica. 17 años, 2012.

"El autobús nos deja lejos de casa a veces porque no puede pasar. La carretera tiene muchos agujeros y a veces se inunda". Varón. 16 años, 2013.

Si observamos los extractos de entrevista, el trabajo de campo nos permite descubrir de forma más concreta cómo ambos contextos chabolistas exteriorizan 
problemas de accesibilidad, características de vivienda y seguridad en sus habitantes. De hecho y en cuanto a la accesibilidad, esta se trata de la posibilidad de encontrar un lugar habitable que esté acorde con los ingresos económicos de las familias. El acceso en ambos lugares está marcado por la ocupación de terrenos privados. En Madrid la accesibilidad es bastante compleja sobre todo en invierno donde se producen inundaciones marcadas por las irregularidades del terreno. El asentamiento está ubicado a 14 kilómetros del centro de la ciudad y existe gran distanciamiento con colegios, institutos, centros médicos, farmacias e incluso supermercados. En Burdeos, es el Ayuntamiento de la ciudad el que asfaltó el terreno para evitar la proliferación de roedores (como se produce en El Gallinero). Thiers-benauge está localizado a tan solo un kilómetro del centro de la ciudad, pero su distanciamiento está marcado por la delimitación del Río Garona que hace de separación dividiendo la ciudad en dos, siendo el margen Sur donde se ubica la mayoría de la población y comercio de la ciudad, y el margen Norte destacado sobre todo por la ubicación de asentamientos y zonas industriales.

En cuanto las características de la vivienda, en ambos asentamientos se levantan viviendas conformadas por chapas, maderas y plásticos. En el caso de Burdeos, la administración local dotó entre 2010 y 2013 de pequeñas casas prefabricadas de madera y letrinas a las familias que se comprometían a ser realojadas en el futuro en edificios y que además mantuvieran a sus hijos en el colegio (evitando por tanto el absentismo escolar y el analfabetismo). En El Gallinero no ha habido letrinas hasta 2015 gracias a la presión de diversos colectivos vecinales y organizaciones no gubernamentales (ONG). Sea en un lugar o en otro, se verifica la ausencia de proyectos que cuenten con labores de mantenimiento de los espacios, es decir, a pesar de dotar con materiales de construcción o letrinas no se tiene en consideración por parte de la Administración que éstos se deterioran con el tiempo y por tanto no se cuenta con partidas presupuestarias que de algún modo mejoren la calidad de las chabolas sobre todo partiendo de la movilidad del colectivo y la presencia de menores de edad.

Por último y en cuanto a la seguridad del entorno, en Madrid el asentamiento se ubica próximo a la Cañada Real Galiana $(1 \mathrm{Km}$.) donde existen elevados niveles de pobreza, exclusión social y venta y distribución de drogas. En Burdeos la situación es distinta ya que la parcela que ubica el lugar está delimitada por un muro que separa de algún modo la ciudad con el asentamiento, lo cual no facilita la integración social dentro de lo urbano.

Según las entrevistas, las demandas específicas de la población hacen constatar cómo un $80 \%$ de la población en ambos asentamientos no quiere vivir en las condiciones expuestas. Sin embargo, el 20\% refleja que prefiere continuar, a pesar de las condiciones, por ser un lugar que aporta seguridad a la propia comunidad y por haber vivido la mayor parte del tiempo tanto en origen, transito como destino en lugares abiertos y en contacto directo con la naturaleza. 


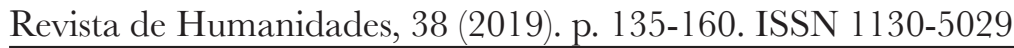

A la vista de los resultados, los medios de subsistencia empleados por la población e incluso los chicos y chicas en los asentamientos son los siguientes:

a. Venta y comercio de ropa y calzado en la propia comunidad.

b. Alquileres de las viviendas cuándo las familias pasan largas temporadas en el exterior.

c. Préstamos entre familias. En este caso con altos intereses en la devolución.

d. Recogida de materiales reciclables como chatarra, cartón y muebles antiguos.

e. Mendicidad.

f. Ayuda de ONG, por ejemplo en vestimenta y alimentación.

Estos medios empleados, sin embargo, crean o mantienen la propia situación de exclusión social y vulnerabilidad en la infancia. En este caso, hemos apreciado lo siguiente:

a. La precariedad del empleo de los progenitores en destino; con horarios incompatibles con la conciliación de la vida familiar, por la escasa renta disponible.

b. Una inserción escolar no adecuada; cuando se produce un desfase curricular entre la formación en origen y en destino; por la ausencia de la familia en el proceso de adaptación al nuevo entorno social y escolar.

c. El aumento de las tasas de abandono escolar (un $70 \%$ a partir de los 16 años) $\mathrm{y}$ absentismo escolar.

d. Aumento de la mendicidad; la cual esta feminizada en ambos espacios observados.

e. Casamiento. El cual sobre todo se lleva a cabo como medio familiar para mejorar las relaciones sociales pero también para recibir la dote en el caso de chicas. Esa aportación económica puede ser en muchos casos de relevante importancia para las familias por la inyección económica o en especies (coches, animales o electrodomésticos).

\subsection{Movilidad familiar y juventud en los asentamientos}

A la luz de los resultados es difícil determinar un número exacto de personas que han transitado por los asentamientos a lo largo del periodo de investigación. Sin embargo, y si nos detenemos en el caso concreto de El Gallinero, el cual es muy próximo en cuanto a variación y periodos a lo analizado en Burdeos, podemos observar lo siguiente: 


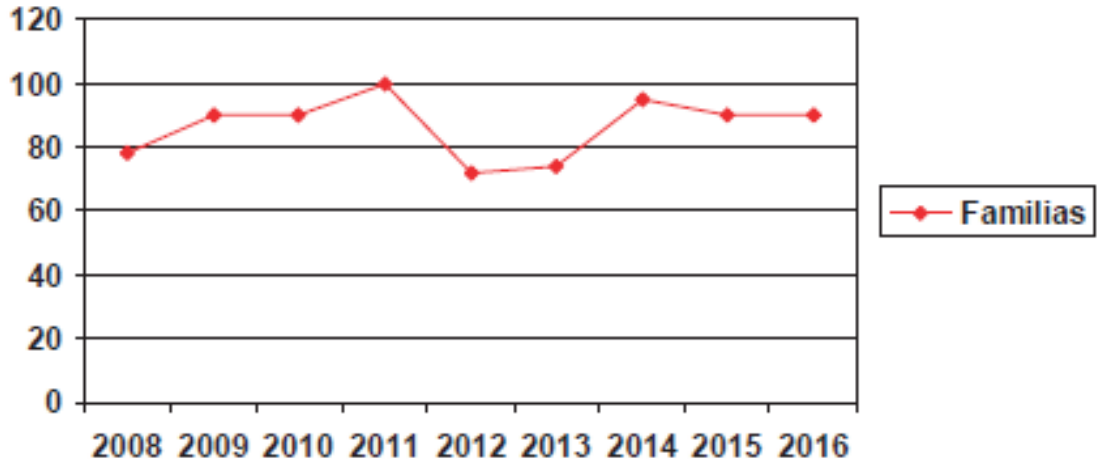

Gráfico 1. Número de familias por año.

Fuente: Gutiérrez, 2017.

Tabla 1. Familiar y población aproximada.

\begin{tabular}{|c|c|c|}
\hline Año & No Familias $^{\circ}$ & Población \\
\hline 2008 & 78 & 386 \\
\hline 2009 & 90 & 440 \\
\hline 2010 & 90 & 404 \\
\hline 2011 & 100 & 600 \\
\hline 2012 & 72 & 321 \\
\hline 2013 & 74 & 390 \\
\hline 2014 & 95 & 435 \\
\hline 2015 & 94 & 400 \\
\hline 2016 & 90 & 400 \\
\hline
\end{tabular}

Fuente: Gutiérrez, 2017.

Tanto el Gráfico 1 como la Tabla 1 nos muestran la evolución de las familias asentadas en el asentamiento de El Gallinero. Los datos reflejan cómo en los años 2012 y 2013 el número de personas desciende. Esto se produce por el aumento de redadas y presión por parte de las administraciones (local y regional) por querer desalojar el lugar, lo cual provocó dos hechos; por un lado parte de la población emigró a otros lugares de España y Europa; y por otro que el número de personas que llegaban al asentamiento no fuera tan progresivo como por ejemplo 2011. Los periodos con más población son 2009, 2011 y 2014. En nuestra investigación evidenciamos como 


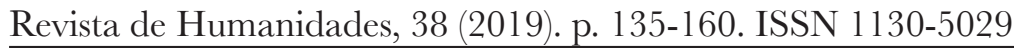

estos años coinciden con expulsiones y desalojos de asentamientos llevados a cabo tanto en Francia como Italia, lo cual provocó un aumento como podemos observar en el caso madrileño, es decir, los flujos de personas se orientan hacia la Península Ibérica.

"Estamos cansados de que la policía intente tirarnos la casa, ¿Adónde vamos a ir? Mi familia piensa en ir a Francia, alli tenemos a mis tíos que seguro que nos ayudan". Varón. 17 años, 2012.

A la vista de las entrevistas, el análisis de las mismas nos manifiestan como la movilidad es interpretada por parte de los jóvenes como oportunidad, enfocada sobre todo a la obtención de un trabajo estable que le permita prosperar dentro de su proyecto individual y familiar. Podemos determinar como la movilidad está marcada en la mayoría de los casos por:

a. La influencia del entorno familiar y comunitario en el país de origen.

b. La posibilidad de encontrar un empleo estable y que permita sustentar a la familia.

c. La movilidad en y entre asentamientos depende en parte de implicación política por resolver los problemas.

d. La movilidad y sus rutas cambian según las condiciones de vida en destino pero también por la presión de los Estados por regular el tránsito y el establecimiento de las personas en asentamientos y áreas vulnerables en las periferias de las grandes ciudades.

Por otro lado, se observa cómo la movilidad se cataliza por las distintas presiones que se ejercen desde las administraciones (regional y local) en el sentido de los continuos desalojos llevados a cabo en el contexto madrileño, los cuales en determinadas ocasiones han vulnerado la Resolución B7-0500 del Parlamento Europeo, sobre la situación del pueblo Romaní en Europa, de 7 septiembre 2010, donde se especifica el derecho a una vivienda digna y la obligación de proveer un alojamiento alternativo apropiado, aunque, como podemos justificar durante nuestra investigación, en el asentamiento se ha vulnerado en repetidas ocasiones entre los años 2010-2012. Enlazado con esta resolución, además se han vulnerado artículos de la Convención sobre los Derechos del Niño, como son: el Art. 16.1; que hace referencia al derecho a la intimidad familiar; el Art. 27; que proclama el derecho a un nivel de vida adecuado y a la protección social en especial respecto a nutrición, vestuario y vivienda; y el Art. 28; que defiende el derecho a la educación y al juego. Aunque estos artículos se desarrollan en diversas materias del derecho, la vulneración de los mismos es confirmada por entidades sociales que operan en el asentamiento.

En el contexto francés, se ha optado por una intervención donde se ha facilitado viviendas a la población pero, sin embargo, se ha optado por reubicar a las comunidades en lugares concretos, manteniendo la política de desalojos y desahucios para todos 
aquellos que han comenzado a ocupar otros terrenos ilegalmente y sin autorización. Por contra, durante el año 2013 hubo intentos por parte del Estado francés de retornar a la población Romaní a origen. En este caso se pagaban los billetes de vuelta y se asignaba a cada persona una cuantía económica que llegaba a ser de aproximadamente $300 €$ para adultos y de $100 €$ para menores de edad. Esta política impactó con una realidad dentro de las fronteras europeas, es decir, la posibilidad de seguir trasladándose por Europa a pesar de que Francia pagara el billete de vuelta y además, de alguna forma, conseguir algo de dinero que servía de ayuda para la familia. Por lo tanto, este tipo de políticas no han frenado la movilidad por Europa de familias procedentes de Rumanía, sino que han fomentado o deslocalizado las rutas Marcu, 2018).

\subsection{Impacto proyectos de intervención}

Durante el trabajo de campo se han observado diversos proyectos implantados en ambos asentamientos. Se han categorizado los datos en torno a tres aspectos destacados y que son relevantes en la vida diaria de la población; educación, vivienda y salud.

En cuanto a la educación, ésta alberga un papel trascendental tanto en el momento presente como futuro de los adolescentes. Los proyectos de intervención que guardan relación con la educación formal (e incluso la no formal) pasan de alguna forma por ser los que más complejidad tienen para perdurar en el tiempo, debido sobre todo, al carácter itinerante de las familias, las necesidades comunitarias por apoyarse en los más jóvenes para obtener recursos, y la falta de compresión por el sistema educacional que existe en el Oeste de Europa en cuanto a la relevancia de la formación para obtener un empleo estable.

En el caso de El Gallinero hubo incluso proyectos de escolarización con adultos para mostrar, de fondo, ejemplo a los más pequeños. Según la Universidad Pontificia Comillas y Save The Children (2014) en el informe La situación de la Infancia en El Gallinero. Los derechos Humanos también son cosa de Niños, se aprecia como para el asentamiento chabolista de El Gallinero, el porcentaje de analfabetos funcionales en la población en edad de trabajar era del $78 \%$. Por otro lado igualmente es destacado nombrar los altos datos de absentismo escolar en ambos contextos, donde la administración se preocupaba más por los datos de escolarizados que por la inclusión social de los jóvenes en las aulas.

En Madrid, la Fundación Imaginario Social por medio del Proyecto Nido operó durante los años 2009-2012 en el asentamiento con la actividad denominada como Cañón, la cual intentaba buscar lugares para que los niños y niñas, adolescentes y jóvenes pudiesen estudiar y, además, conseguir mejores resultados académicos en la escuela. Para ello se sirvió de actividades paralelas como de Fútbol, Danza y 


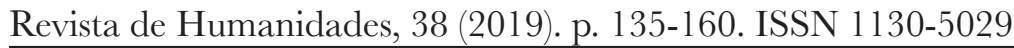

Campamentos, como medio de fomentar el entusiasmo y focalizar un objetivo. Ésta entidad junto con Cruz Roja lograron en 2013 escolarizar a todos los niños y niñas menores de 16 años, con financiación local y regional.

"Nos encanta jugar al futbol. Salimos de aqui y después nos podemos duchar. Queremos hacer un equipo que juegue con gitanos españoles”. Varón. 15 años, 2013.

En cuanto a Burdeos, desde 2013 hasta la actualidad se ha optado por otro tipo de intervenciones en las cuales se integraba a los padres y madres (Clave-Mercier y Olivera, 2018). La Administración Local establecía un contrato social con las familias, donde se acordaba mantener a los niños y niñas en el colegio mientras los padres recibían ayudas económicas materializadas en especies (ayudas transporte, una casa portátil de madera e incluso búsqueda y asesoramiento de empleo).

Por último, en ambos asentamientos, el 80\% de los adolescentes entrevistados mostraban carencias a la hora de tener una alimentación apropiada, lo que se traducía en acudir a la escuela sin desayunar, perturbando por tanto al rendimiento y a atención en el aula. Además la gran cantidad de redadas nocturnas efectuadas por la Policía en los asentamientos, empeoraba aún más la situación originando por tanto somnolencia y déficit de atención.

"La Policía viene de noche y asustan a los niños. Muchas veces recibimos golpes. Solo quieren darnos miedo para que nos vayamos de aquí, esto no es normal". Mujer. 40 años, 2011.

En cuanto a los proyectos orientados a satisfacer las necesidades de vivienda, éstos han sido los que menos resultados han obtenido en Madrid debido al asentamiento en un terreno privado y por la falta de compromiso de las administraciones por reubicar a la población en otras zonas de la capital. En este caso y como hemos evidenciado, eran las entidades sociales, parroquias y voluntarios los que asumían sobre todo las ayudas a las familias. Durante 2010-2012 y debido a la gran cantidad de desalojos y desahucios, las parroquias ofrecían sus espacios hasta cuando se encontrara un lugar donde residir. En este sentido podemos destacar el proyecto de intervención que las Parroquias de San Carlos Borromeo y Santo Domingo de la Calzada, denominado "Tenemos un Plan" en 2012. Este proyecto tomaba como base la intervención desde el acceso a la vivienda, como forma de hacer que la población de estudio pudiera integrarse socialmente en la Comunidad de Madrid. Este proyecto intentaba, bajo el trabajo constante de la familias, dar solución a problemas como: autoconstrucción espontánea, insalubridad, precariedad, materiales deleznables, marginalidad social, hacinamiento, vulnerabilidad, degradación ambiental, falta de equipamiento y empleo (Tenemos un Plan, 2012). Finalmente el proyecto no llegó a materializarse por falta de compromiso e inversión, sin embargo, es oportuno reflejar por medio de las siguientes figuras la propuesta del proyecto. 
Figura 1. Ordenación Gallinero según el proyecto “Tenemos un Plan”. Año 2012.
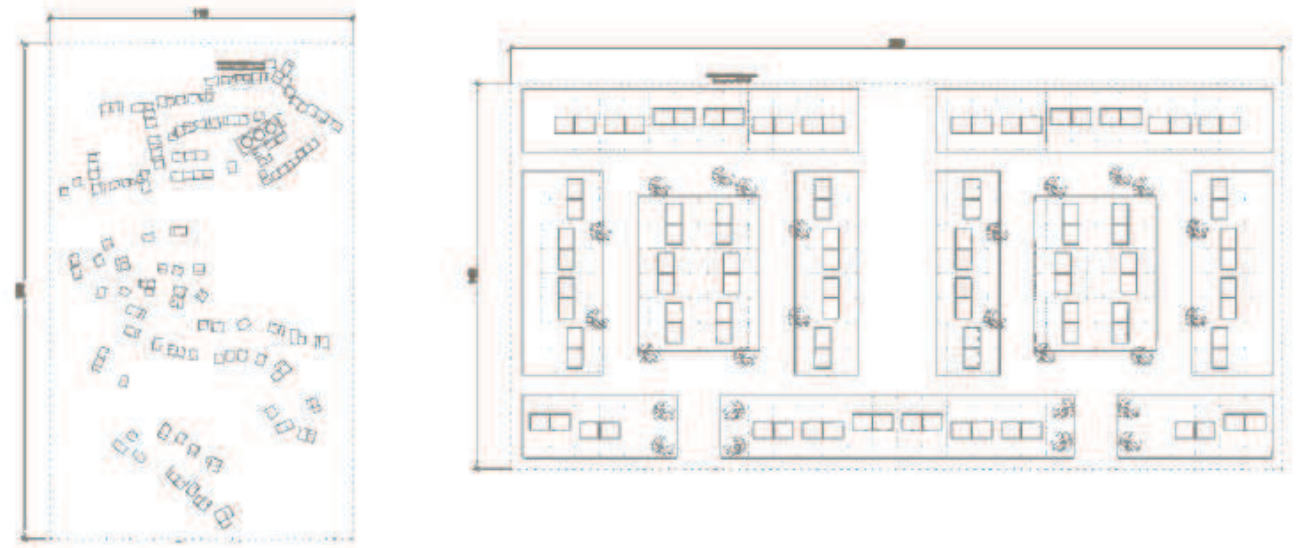

Fuente: Proyecto “Tenemos Un Plan”. 2012

En esta imagen podemos observar la propuesta del Proyecto. A la izquierda se observa el Gallinero en 2011 en una superficie aproximada de 20.000 metros cuadrados y a al derecha la propuesta de ubicar el asentamiento en una mayor de 40.000. Por otro lado el proyecto integraba una inversión en mejorar las viviendas (con materiales más resistentes) y la integración de letrinas.

Por último, y en relación a los proyectos orientados a salud, se observan diferencias entre las intervenciones desarrolladas en los asentamientos. Por un lado en Burdeos se interviene por medio de la ONG Médicos del Mundo a través de programas de ayuda humanitaria donde se registran los usuarios y se mantiene un cierto control en el caso de observar enfermedades. La intervención pública, en este caso, queda a un segundo nivel. La privatización del servicio resulta ser una vía más económica para las administraciones a pesar de estar tratando con población de otros países de la Unión Europea.

En el caso de Madrid, es la Comunidad de Madrid la que por medio de su Consejería de Salud se intervenía en la zona. El equipo contaba con ambulancia, enfermero y médico. Se atendía a la población casi diariamente, se derivaban a especialistas y se hacían más controles y campañas como por ejemplo de parásitos, necesidades oculares, intervenciones específicas con bebés y de prevención de enfermedades de transmisión sexual (ETS). En este caso la Administración ocupaba un papel relevante para la igualdad de condiciones y solidaridad entre países comunitarios.

Hay que tener en cuenta, que ni en El Gallinero ni en Thiers-benauge se detectaron enfermedades distintas a las de la población que vivía en las ciudades. En este sentido, se verifica y por tanto se anula el falso mito de que la población romaní transporta enfermedades y las contagia (Gutiérrez, 2015). 


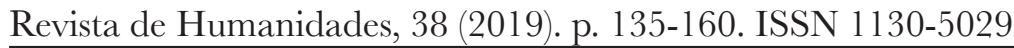

Por último y en relación a entidades sociales, se ha evidenciado como la capacidad económica de las mismas es un aspecto trascendental en el desarrollo de las intervenciones, en primer lugar por la escasa dotación económica que se destina (por parte del sector público) para resolver problemas de inclusión social, mejoras en hábitat o mantenimiento de servicios sanitarios y escolares (como por ejemplo rutas de autobús). Las dotaciones suelen depender, en muchos casos, de la implicación política. Esto afecta directamente en el mantenimiento de los proyectos cuando existen cambios de gobierno, por lo tanto, los proyectos pueden llegar a sufrir recortes, terminan suspendiéndose o no llegan a consolidarse y realizarse evaluaciones de cómo ha transcurrido a lo largo del tiempo.

"No podemos dejar que los proyectos sea asumidos por entidades privadas que solo piensan en ganar dinero y no en los propios beneficiarios. En los concursos públicos, los proyectos se suelen conceder a entidades que hacen la misma tarea por menosyeso al final se traduce en pan para hoy y hambre para mañana”. Trabajadora Social. 30 años, 2014.

"Si las Administraciones no nos conceden ayudas o bien no nos facilitan el dinero a tiempo para poder llevar a cabo los proyectos, nos vemos en la tesitura de no pagar a nuestros trabajadores". Presidenta ONG. 62 años, 2011.

Cómo podemos ver en los extractos de entrevista, existe una dependencia considerable de algunas entidades a las administraciones, es decir, éstas no pueden implantar proyectos en su totalidad si existen retrasos de pagos y financiación, o bien ciertas entidades tienen que cesar su trabajo porque no han recibido subvenciones o se recortan los presupuestos.

Por último, la contratación de personal especializado en contextos de riesgo e inclusión social de personas vulnerables, es también un hándicap para la perfecta implantación de los proyectos. Por un lado no se reconoce el trabajo profesional con sueldos dignos, estando como hemos evidenciado, personal contratado durante 40 horas semanales cobrando $800 €$ y exigiendo un conocimiento del colectivo absoluto. De otro lado, ha sido normalizado el carácter voluntario en la acción social, lo cual deslegitima a los profesionales en determinadas áreas, ya que los voluntarios pasan a realizar trabajos con una gran implicación. Se evidenció como voluntarios han llegado a pasar más horas que técnicos contratados y en numerosos casos $(70 \%)$ han desempeñado tareas que exigen un profesional cualificado ante situaciones de emergencia como desalojos, desahucios, redadas, denuncias, urgencias sanitarias o bien realizar un contrato social o diagnóstico social.

"No puedo vivir en Madrid ganando 800€, es imposible. No se reconoce nuestro trabajo”. Educador Social. 25 años, 2011.

"Soy voluntaria y me encanta pero he de reconocer que en determinadas tareas, paso más tiempo en El Gallinero que los propios trabajadores”. Voluntaria. 40 años, 2010. 


\section{CONGLUSIONES}

Teniendo en cuenta los planteamientos del estudio, concluimos que, tras analizar las experiencias en los asentamientos chabolistas de El Gallinero y Thiers-benauge, se confirma la relevancia en la implantación de un modelo de Trabajo Social de corte comunitario caracterizado por contener estrategias de intervención innovadoras y por saber incluir en sus propios proyectos y programas a la población con el interés de que ellos mismos sean parte de su propio desarrollo.

Las entrevistas han revelado que los adolescentes e incluso sus familias incluyen la movilidad geográfica como estrategia ante problemas económicos, desempleo de larga duración y continuación de una formación. Esta movilidad unida a la exclusión social que vive el colectivo incluso desde origen, facilitan de algún modo el asentamiento en áreas vulnerables y periféricas en las ciudades, donde se desarrollan puntos chabolistas.

Estos resultados, son muy similares a los encontrados en otros estudios como el llevado a cabo por Marcu (2018) donde se ha analizado las razones por las que los jóvenes deciden emprender una movilidad dentro de Europa y coincide con nuestro estudio dado que los jóvenes están interesados en encontrar mejoras económicaslaborales. No obstante, sería pertinente reconstruir las etapas laborales previas al asentamiento en espacios concebidos como de riesgo, además de averiguar la incidencia que han tenido los procesos políticos y estructurales en origen hacia el colectivo romaní de tal modo que puedan interpretarse aquellos aspectos que puedan incidir sobre la decisión última de comenzar el proceso de movilidad. Por otro lado, las propias costumbres de la población gitano rumana por su carácter itinerante, chocan con aquellas políticas públicas de reubicación y mantenimiento en hogares estanco, es decir, se produce un conflicto de intereses en cuanto cómo debe vivir una persona, sobre todo por el control social que se lleva a cabo por la mayoría de países europeos.

Cabe resaltar cómo las políticas públicas de corte social y orientadas a la inclusión social, se caracterizan sobre todo por su complejidad y debilidad, es decir, por las deficientes o carentes estructuras y redes entre administraciones y entre entidades sociales, lo cual a la hora de incidir en la inclusión social de los Rrom no logran incluir socialmente a la población o mejorar su calidad de vida. Sería pertinente por tanto mejorar los diagnósticos elaborados y orientar objetivos más específicos tanto a corto como largo plazo. A su vez a nivel europeo, sería interesante mantener una política común de atención a la personas de movilidad elevada y que se ubica en entornos como los que hemos observado, ya que desde esta posición puede mejorar, en cierto modo, a mejorar la gestión de los recursos y poder atender igualitariamente a ciudadanos europeos con recursos bajos.

Hasta donde revelan las entrevistas, habría que analizar el papel de los Estados (español y francés) en relación a qué medidas se adoptan dentro de la intervención 


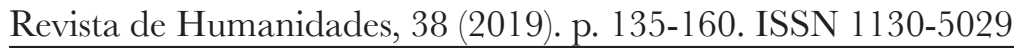

con familias de gran movilidad, es decir, qué planes y programas se ponen en marcha para afrontar el establecimiento de las familias en entornos desfavorecidos y, además, cómo éstos espacios afectan a los adolescentes dentro del cambio evolutivo y de desarrollo que se viven a nivel cognitivo y físico y que es propio de la adolescencia.

Por último, cabe distinguir, que una formación adaptada a los adolescentes constituye la base más relevante para que ellos mismos construyan aprendizajes que puedan generar una continuidad en su proceso de formación y su desarrollo profesional y vital. Sería pertinente valorar el desarrollo de la formación profesional en adolescentes como medio de contribuir a la adquisición de una formación reglada, pero además de permitir que el colectivo pueda encontrar un trabajo específico, evitando de tal modo la probabilidad de manifestar actitudes delictivas o de mendicidad.

Otro aspecto que se ratifica en diversas investigaciones, tales como (ClaveMercier y Olivera, 2018; Marcu, 2018), es el impacto que tienen territorios con gran carga de vulnerabilidad social sobre los adolescentes, además de, falta de acceso a recursos públicos, degradación ambiental, condiciones de habitabilidad muy precarizados, deterioro en las relaciones de convivencia (tanto dentro como fuera de la comunidad), altos niveles de pobreza y exclusión social y elevados índices de desempleo.

Asimismo, se interpreta como necesario seguir investigando en aras de descubrir si los diversos proyectos de intervención implantados en contextos como los estudiados, proporcionan cambios evidentes en la población y capacitan la incorporación laboral, la cual puede desarrollar nuevas vías inclusivas en la sociedad. Todo ello enriquecería valiosamente los resultados obtenidos en esta investigación.

Para finalizar, como limitaciones de la presente investigación, podemos señalar la complejidad existente en la recogida de información debida, sobre todo, por las características propias de los sujetos, el difícil acceso a los asentamientos y la creación con el tiempo de confianza recíproca (entre investigador y población) para poder extraer información.

\section{REFERENGIAS BIBLIOGRÁFICAS}

Baldoni, E. (2012). Los "campamentos nómadas" en Italia, entre marginalización y negación de derechos. OBETS, Revista de Ciencias Sociales, 7(2), 167-183.

Bauman, Z. (2006). Modernidad líquida. Buenos Aires: Fondo de Cultura Económica.

Beck, U. (1992). Risk society. Londres: SAGE Publications. https://doi. org/10.4135/9781412952552.n244

Bontempelli, S. (2015). L 'invenzione degli Zingari. La questione Rom tra Antiziganimo, Razzismo ed Etnicizzazione. Iperstoria, Testi Letterature Linguaggy, 6, 45-56. 
Jóvenes romaníes en asentamientos chabolistas... - J.D. Gutiérrez y C. Gimeno

Brearley, M. (2001). The Persecution of Gypsies in Europe. American Behavioral Scientist, 45(4), 588-599.

Briggs, D. (2016). Nuevos "extranjeros" y nuevos miedos: Las barreras culturales y estructurales para la juventud trabajadora rumana en Londres. Revista de Estudios de Juventud, 113, 103-119.

Castel, R. (1995). De la exclusión como estado a la vulnerabilidad como proceso. Archipiélagos, Cuadernos de Crítica de la Cultura, 21, 27-36.

Clave-Mercier, A. y Olivera, M. (2018). Inclusion and the 'Arts of Resistance'. How Do Roma Migrants Develop Autonomy in the Context of Inclusion Policies? Intersections. East European Journal of Society and Politics, 4(2), 149-168.

Climent, V. (2006). Sociedad del riesgo: producción y sostenibilidad. Revista Papers, 82, 121-140. https://doi.org/10.5565/rev/papers.2052

Universidad Pontificia Comillas y Save The Children (2014). La situación de la Infancia en El Gallinero. Los Derechos Humanos también son cosa de niños. Madrid: Informe y Publicaciones.

Cresswell, T. (2006). On the Move: Mobility in the Modern WesternWorld. London: Routledge.

Csepeli, G. y Simon, D. (2004). Construction of Roma identity in Eastern and Central Europe: perception and self - identification. Journal of Ethnic and Migration Studies, 30(1), 129-150, DOI: 10.1080/1369183032000170204

Dahrendorf, R. (1979). Life Chances. Chicago: Uniersity of Chicago Press.

Dell'Agnese E. \& Vitale T. (2007). Rom e sinti: una galassia di minoranze senza territorio. In Amiotti G., Rosina A. (Eds.), Tra identità ed integrazione: passato e presente delle minoranze nell'Europa mediterranea. Milán: Franco Angeli

Di Noia, L. (2016). Radici storiche e processi sociali dell'esclusione dei ROM. Società e Transformazioni Sociali, 4, 21-55.

Diminescu, D. (2004). La mobilité des jeunes romains a l'heure de l'elargissement européen. Hommes et Migrations, 1251, 45-50.

Dovigo, F. (2014). El tratamiento de la diversidad en las instituciones educativas. En J. Gairín, (Coord.) Colectivos Vulnerables en la Universidad. Reflexiones y

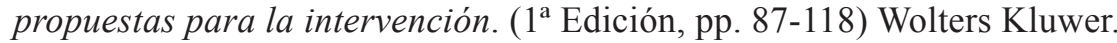

EAPN (2017). El estado de la Pobreza: Seguimiento del indicador de riesgo de pobreza y exclusión social en España (2008-2016). Informes de la Red Europea de Lucha contra la Pobreza y la Exclusión Social.

Empez, N. (2009). The fieldworker as a social worker: dilemma's in research with Moroccan Unaccompanied minors in Spain. En I. van Liempt y V. Bilger (ed.). The Ethics of Migration Research Methodology. Dealing with Vulnerable Immigrants. Eastbourne (Gran Bretaña): Sussex Academic Press. 
Revista de Humanidades, 38 (2019).p. 135-160. ISSN 1130-5029

Ferrera, M. (2018). Existe más solidaridad de lo que parece: retos y posibilidades de la Europa social. En G. Jaraíz (ed.) Bienestar Social y Políticas Públicas: Retos para pactar el futuro (111-134). Madrid: Los Libros de la Catarata.

Ferrera, M. (2005). The Boundaries of Welfare. European Integration an the new Spatial Politics of Social Protection. Oxford: Oxford University Press.

Friedlander, W.A. (1977). Dinámica del Trabajo Social. México: Editorial Pax.

Gaitán, L. (2003). Ciudadanía, Participación y Trabajo Social. En Ponencia presentada en Inauguración del Curso Académico 2003/2004 de la Escuela de Trabajo Social de la Universidad de Murcia.

Gimeno, Ch. y Gutiérrez, J.D. (2019). Fostering unaccompanied migrating minors. A cross-border comparison. Children and Youth Services Review, 99, 36-42.

Gimeno, Ch. (2013). Menores que migran solos y sistemas de protección a la infancia. Zerbitzuan, Revista de Servicios Sociales, 53, 109-122.

Gobierno de España (2011). Programa Nacional de Reformas. Informes Ministerio de Sanidad, Servicios Sociales e Igualdad.

Griffin, C. (2008). Nomads under the Westway Irish Travellers, Gypsies and other traders in west London. Hertfordshire: University of Hertfordshire Press.

Grill, J. (2012). Going up to England. Exploring mobilities among Roma from Eastern Slovakia. Journal of Ethnic and Migration Studies, 38 (8), 1269-1287.

Gutiérrez, J.D. (2017). Movilidad del Pueblo Gitano de Europa del Este en el asentamiento chabolista de El Gallinero. Anduli. Revista Andaluza de Ciencias Sociales, $\mathrm{N}^{\circ}$ 16, 73-89.

Gutiérrez, J.D. (2017a). Imaginarios sociales de la itinerancia en dentro de la diáspora gitana. Imagonautas. Revista Interdisciplinaria sobre imaginarios sociales, 9, 40-57.

Gutiérrez, J.D. (2015). Los menores gitanos rumanos de "El Gallinero": Etapas de desarrollo en un contexto de riesgo. Revista Electrónica de Investigación y Docencia (REID), 13, 27-44.

Gutiérrez, J.D. y Diz, J. (2017). Cooperación Internacional en Marruecos: empoderamiento para insertar socio-laboralmente a jóvenes en contextos de riesgo. Cuadernos de Trabajo Social, 30 (1), 163-174.

Gutiérrez, J.D. y Ortega, J. (2019). Educación y movilidad de la comunidad Rom en Madrid: el caso del Gallinero. Revista de Estudios Socioeducativos (RESED), 7, $154-165$.

Harper, K., Steger, T. y Filcak, R. (2009). Environmental Justice and RomaCommunities in Central and Eastern Europe. Environmental Policy and Governance, 19, 251-268.

Humphris, R. and Sigona, N. (2016). Mapping unaccompanied asylum seeking children in England. En Becoming Adult Research Brief Series, N ${ }^{\circ}$ 1. London: UCL 
Jóvenes romaníes en asentamientos chabolistas... - J.D. Gutiérrez y C. Gimeno

Kohli, R.K.S (2014). Proteger a los menores en situación de migración independiente. En Jiménez, A.S., Pantoja, A., Leiva, J.J. y Moreno, E. (Coords.). Infancia en contextos de riesgo. XXV Años de la Convención sobre los Derechos del Niño. Granada: GEU Editorial.

Kohli, R.K.S. (2006). The comfort of strangers: Social work practice with unaccompanied asylum seeking children and young people in the UK. Child and Family Social Work, 11, 1-10. https://doi.org/10.1111/j.1365-2206.2006.00393.x

Krmpotic, C. S. (1999). El concepto de Necesidad y políticas de Bienestar. Argentina: Espacio Digital.

Las Heras, P. (1999, 17 Julio). Rumanos en Madrid. $A B C$. Recuperado de hemeroteca. abc.es/nav/Navigate.exe/.../madrid/abc/1999/07/17/064.html

López-Catalán, O. (2014). Piedra, Papel y Tijera: vivienda y gestión del asentamiento de la población rrom gitana rumana en el Área Matropolitana de Barcelona (20062014). Revista Andaluza de Antropología, 7, 102-129.

López-Riopedre, J.L. (2017). Migraciones "Al margen": grupos rumanos, diversidad y control social. Revista Internacional de Estudios Migratorios, 7(4), 229-256.

Luhmann, N. (1991). Sistemas Sociales: lineamientos para una teoría general. México D.F.: Alianza Editorial.

Marchioni, M. (1999). Comunidad, participación y desarrollo. Teoría y metodología de la intervención comunitaria. Madrid: Editorial Popular.

Marchioni, M. (1989). Planificación social y organización de la comunidad. Alternativas avanzadas a la crisis. Madrid: Editorial Popular.

Marcu, S. (2018). Geografias de la movilidad humana en el siglo XXI: jóvenes de la Europa del Este en España. Madrid: Ediciones Complutense.

Marcu, S. (2013). La movilidad transfronteriza de rumanos en España en tiempos de crisis. Revista Internacional de Sociología (RIS), 71, (1), 115-141.

McNeil, E.B. (1954). Community Organization of Social Welfare. En American Association of Social Workers. Social Work Year Book. Nueva York: AASW.

Moix, M. (1991). Introducción al Trabajo Social. Madrid: Trivium.

Pajares, M. (2007). Inmigrantes del Este. Procesos Migratorios de los rumanos. Barcelona: Icaria Antrazyt.

Parlamento Europeo (2007). El Tratado de Lisboa. Fichas Técnicas sobre la Unión Europea. Descargado de: http://www.europarl.europa.eu/ftu/pdf/es/FTU_1.1.5.pdf

Pastor, E. (2015). Social Work and Local Community Development in 21 Century. Arbor, 191 (771), 1-17. doi: http://dx.doi.org/10.3989/arbor.2015.771n1010

Pastor, E. (2004). La participación ciudadana en el ámbito local, eje transversal del trabajo social comunitario. Alternativas: Cuadernos de Trabajo Social, 12, 103-138.

Pastoor, L. D. W. (2015). The mediational role of schools in supporting psychosocial transitions among unaccompanied young refugees upon resettlement in Norway. 
Revista de Humanidades, 38 (2019).p. 135-160. ISSN 1130-5029

International Journal of Educational Development, 41, 245-254. https://doi. org/10.1016/j.ijedudev.2014.10.009.

Picornell, A. (2015). Un acercamiento a la práctica comunitaria del Trabajo Social en defensa de la ciudadanía de la infancia: la experiencia REDidi. Interacción y Perspectiva. Revista de Trabajo Social, 1 (5), 73-84.

Proyecto Nido (2011). Todos los niños/as tienen derecho a una educación que asegure su integración social”. Madrid: Fundación Imaginario Social.

Purcell, K. (2010). Flexible employment, student labour an the changing structure of the UK labour market in university cities. Centro de Estudios da Metropole Texto para discussao No.007

Reyniers, A. (1995). «Gypsy population and their movement within Central an Eastern Europe and towards some OECD countries». En International Migration an Labour Market Policies Occasional Papers, 1, pp. 12.

Ryan, T. (1996). Risk Management and People with Mental Health Problems. H. Kemshall \& J.Pritchard (eds.), Good Practice in Risk Assessment and Risk Management, 1. Londres: Jessica Kigsley.

Sheller, M. y Urry, J. (2006). The new mobilities paradigm. Environment and Planning A, 38, 207-226.

Stevenson, O. (1999). Old People at Risk. Social Work, 36. Londres: Jessica Kingsley.

Twelvetrees, A. (1985). Community Work. Londres: Macmillan Press.

Vassilev, R. (2004). The Roma of Bulgaria: A pariah minority. The Global Review of Ethonopolitics, 3(2), 40-51.

Vigh, H.E. (2006). Navigating Terrains of War: Youth and Soldiering in Guinea Bissau. New York: Berghahn Books.

Viruela, R. (2010). Movilidad geográfica de los rumanos (Estructura territorial de las migraciones interiores en España) Revista de Metodología de Ciencias Sociales, Empiria, 19, 157-181.

Warner, F. (1992). Introduction. Risk: Analysis, Perception and Management Report of a Royal Society Study Group. Londres: Royal Society. 